\title{
Variability Analysis based on POSS1/POSS2 Photometry
}

\author{
Areg M. Mickaelian ${ }^{1}$, Alain Sarkissian ${ }^{2}$ and Parandzem K. Sinamyan ${ }^{1}$ \\ ${ }^{1}$ Byurakan Astrophysical Observatory (BAO), Byurakan 0213, Aragatzotn Province, Armenia \\ email: aregmick@aras.am, spk7711@gmail.com \\ ${ }^{2}$ Laboratoire Atmosphères, Milieux et Observations Spatiales, 78280 Guyancourt, France \\ email: alain.sarkissian@latmos.ipsl.fr
}

\begin{abstract}
We introduce accurate magnitudes as combined calculations from catalogues based on accurate measurements of POSS1- and POSS2-epoch plates. The photometric accuracy of various catalogues was established, and statistical weights for each of them have been calculated. To achieve the best possible magnitudes, we used weighted averaging of data from APM, MAPS, USNO-A2.0, USNO-B1.0 (for POSS1-epoch), and USNO-B1.0 and GSC 2.3.2 (for POSS2-epoch) catalogues. The r.m.s. accuracy of magnitudes achieved for POSS1 is 0.184 in $B$ and 0.173 mag in $R$, or 0.138 in $B$ and 0.128 in $R$ for POSS2. By adopting those new magnitudes we examined the First Byurakan Survey (FBS) of blue stellar objects for variability, and uncovered 336 probable and possible variables among 1103 objects with POSS2-POSS1 $\geqslant 3 \sigma$ of the errors, including 161 highly probable variables. We have developed methods to control and exclude accidental errors for any survey. We compared and combined our results with those given in Northern Sky Variability Survey (NSVS) database, and obtained firm candidates for variability. By such an approach it will be possible to conduct investigations of variability for large numbers of objects.
\end{abstract}

Keywords. techniques: photometric, methods: statistical, stars: variables: other, stars: earlytype, cataclysmic variables, white dwarfs, BL Lacertae objects: general

\section{POSS1/POSS2 Best Magnitudes and Variability}

Variability is one of the key parameters for investigating stellar physics and evolution; the discovery and study of variable stars and understanding their changes is therefore extremely important. However, there are only 80,671 known variables listed in the General Catalogue of Variable Stars (GCVS; Samus et al. 2011), and compared to the total of $\sim 1$ billion stars with available photometric data is is of course a rather small number. Accurate photometry is needed to compare brightness and reveal candidate variables. Photometric catalogues of the required accuracy contain relatively small numbers of objects, and cannot be used to check the possible variability of any object over the whole sky. More than 1 billion objects have been measured photometrically from the Palomar Observatory Sky Surveys (POSS) epochs 1 and 2: (1948-1958 and 1986-2000, respectively) and their Southern Sky extensions at ESO and AAO, together covering the whole sky and at present giving $\sim 0.2^{m}-0.3^{m}$ r.m.s. photometry for objects down to at least $\sim 21^{m}$. In this paper we use "POSS1" and "POSS2" to refer to the whole sky. The data are given in United States Naval Observatory catalogue (USNO-A2.0; Monet et al. 1998), Automated Plate Measurement catalogue (APM; McMahon et al. 2000), Minnesota Automated Plate Scanner catalogue (MAPS; Cabanela et al. 2003), USNO-B1.0 (Monet et al. 2003), and the HST Guide Star Catalogue (GSC 2.3.2; Lasker et al. 2008). As those catalogues are all based on the same observations (POSS1 and POSS2 photographic plates), we may expect to derive similar results for POSS1 and POSS2 photometry, respectively. 
Table 1. Photometric accuracy and statistical weights of individual catalogs.

\begin{tabular}{|c|c|c|c|c|c|c|}
\hline Catalogue & Epoch & $\begin{array}{c}\text { Photometric } \\
\text { bands }\end{array}$ & $\begin{array}{r}\text { Number of } \\
\text { objects }\end{array}$ & $\begin{array}{c}\text { Catalogues } \\
\text { r.m.s. }\end{array}$ & r.m.s.B/weight & r.m.s.R/weight \\
\hline $\begin{array}{l}\text { MAPS } \\
\text { APM } \\
\text { USNO-A2.0/B1.0 } \\
\text { USNO-B1.0 } \\
\text { GSC } 2.3 .2 \\
\text { Tycho-2 } \\
\text { SDSS } \\
\text { 2MASS } \\
\text { GCVS } \\
\text { NSVS }\end{array}$ & \begin{tabular}{|c|} 
POSS1 \\
POSS1 \\
POSS1 \\
POSS2 \\
POSS2 \\
$1989-1993$ \\
$2000-2008$ \\
$1997-2001$ \\
$1900-2011$ \\
$1999-2000$
\end{tabular} & \begin{tabular}{|c|}
$\mathrm{O}, \mathrm{E}$ \\
$\mathrm{b}, \mathrm{r}$ \\
$\mathrm{B} 1, \mathrm{R} 1$ \\
$\mathrm{~B} 1, \mathrm{R} 1, \mathrm{~B} 2, \mathrm{R} 2, \mathrm{I}$ \\
$\mathrm{j}, \mathrm{V}, \mathrm{F}, \mathrm{N}$ \\
$\mathrm{BT}, \mathrm{VT}$ \\
$\mathrm{u}, \mathrm{g}, \mathrm{r}, \mathrm{i}, \mathrm{z}$ \\
$\mathrm{J}, \mathrm{H}, \mathrm{Ks}$ \\
V or other \\
R (VROTSE)
\end{tabular} & \begin{tabular}{|r|}
$89,234,404$ \\
$166,466,987$ \\
$526,280,881$ \\
$1,045,913,669$ \\
$945,592,683$ \\
$2,539,913$ \\
$357,000,000$ \\
$470,992,970$ \\
80,671 \\
$14,000,000$
\end{tabular} & $\begin{array}{c}0.2-0.3 \\
0.5 \\
0.3 \\
0.3 \\
0.13-0.22 \\
0.01-0.10 \\
0.03 \\
0.02 \\
0.1 \\
0.04\end{array}$ & $\begin{array}{l}0.308 / 0.409 \\
0.413 / 0.228 \\
0.327 / 0.363 \\
0.206 / 0.414 \\
0.173 / 0.586\end{array}$ & $\begin{array}{l}0.271 / 0.389 \\
0.318 / 0.283 \\
0.295 / 0.328 \\
0.153 / 0.408 \\
0.127 / 0.592\end{array}$ \\
\hline $\begin{array}{l}\text { Combined POSS } 1 \\
\text { Combined POSS } 2\end{array}$ & \begin{tabular}{|l|}
$1948-1958$ \\
$1986-2000$
\end{tabular} & $\mid \begin{array}{c}\mathrm{O} / \mathrm{b} / \mathrm{B} 1, \mathrm{E} / \mathrm{r} / \mathrm{R} 1 \\
\mathrm{~B} 2 / \mathrm{j}, \mathrm{R} 2 / \mathrm{F}\end{array}$ & & & $\begin{array}{l}0.184 \\
0.138\end{array}$ & \begin{tabular}{|l}
0.173 \\
0.128
\end{tabular} \\
\hline
\end{tabular}

Table 2. Variability classes and the numbers based on POSS2/POSS1 comparison.

\begin{tabular}{|l|c|r|r|r|}
\hline Variability classes & POSS2/POSS1 & $\mathbf{B}$ & $\mathbf{R}$ & $\mathbf{B}+\mathbf{R}$ \\
\hline Extremely variable & $\geqslant 10.00$ & 15 & 9 & 1 \\
Strongly variable & $5.00-9.99$ & 46 & 52 & 28 \\
Probable variable & $3.00-4.99$ & 115 & 101 & 132 \\
Possible variable & $2.00-2.99$ & 200 & 177 & 175 \\
\hline All objects & $\geqslant \mathbf{2 . 0 0}$ & $\mathbf{3 7 6}$ & $\mathbf{3 3 9}$ & $\mathbf{3 3 6}$ \\
\hline
\end{tabular}

Unfortunately, during automatic object identification and measurements on different plates, accidental errors can happen, and automatic cross-matching then leads to erroneous results. To derive best magnitudes from all the POSS1 and POSS2 measurements, we

- corrected for systematic differences between MAPS-APM-USNO-A2.0/B1.0 and USNO-B1.0-GSC 2.3.2 magnitudes,

- combined POSS1-based magnitudes (MAPS, APM, USNO) onto POSS1-best,

- combined POSS2-based magnitudes (USNO and GSC 2.3.2) onto POSS2-best,

- eliminated accidental errors (measurements with large individual errors),

- established statistical weights for each catalogue:

MAPS : APM : USNO = $0.409: 0.228: 0.363$ and USNO $:$ GSC $=0.414: 0.586$,

- calculated $\mathrm{O} / \mathrm{b} / \mathrm{B} 1, \mathrm{E} / \mathrm{r} / \mathrm{R} 1$ and $\mathrm{B} 2 / \mathrm{j}, \mathrm{R} 2 / \mathrm{F}$ by using the derived statistical weights.

Table 1 gives the previous and newly-obtained photometric accuracy and statistical weights of individual catalogues, together with the accuracy for the combined POSS1and POSS2-based magnitudes, which we call POSS1-best and POSS2-best.

We have also derived transformation formulæ from POSS2 to POSS1, POSS to Johnson $B$ and $V$, POSS to SDSS $g$ and $r$, and POSS- $R$ to NSVS- $R$. The differences between POSS2-best and POSS1-best then reveal objects which are variables. Table 2 summarizes our counts and classifications of those objects.

There were 294 NSVS variables among those objects. The 336 objects which we newly identified as variable included 37 that were already in the NSVS, so our analysis of our analysis of POSS1/POSS2 photometry has identified 299 new possible variables. 


\section{Multiwavelength Data and the Physical Nature of Objects}

Possible variable objects among FBS Blue Stellar Objects (BSOs) might include blazars (large variability, radio, X-ray, polarization), QSOs/Seyferts (small variability), CVs (DN, NL, etc.; optical variability, X-ray), WDs (pulsating WDs, ZZ Ceti variables), flare stars (dMe: dwarf M stars at flare stage), and some others. Cross-correlation with radio and X-ray catalogues and proper-motion data revealed:

- 19 radio/IR variable sources (18 are radio and 6 are IR sources). Of those, 12 are extragalactic objects (11 AGN and 1 galaxy) and 2 are planetary nebulae (PN). One source appears to be an unknown object and 4 are associated with stars (WDs and subdwarfs; chance associations with large positional differences).

- 36 X-ray sources having variability (9 of them are the same radio AGN). Out of those, 21 are AGN, including 9 radio sources (among them 3 blazars). But X-ray is also useful for identifying CVs and WDs; 5 CVs and 8 variable WDs have thus been identified, while 2 objects have no classification but are also probable AGN/CV/WD.

- Altogether, 46 out of 336 variable FBS BSOs are either radio, IR or X-ray sources. Among the other 290 objects 4 are AGN, 7 are other galaxies, 10 are CVs, 53 are WDs, 66 are subdwarfs, 7 are NHB/HBB, 26 are stars without a classification, and 117 are unknown objects. We have thus established the variability of 336 objects, including 117 of unknown nature.

- If we take only the 161 objects with extreme/strong/probable variability, then the total numbers are 13 AGN, 4 other galaxies, 1 PN, 9 CVs, 27 WDs, 32 subdwarfs, 6 NHB/HBB, 11 stars without a classification, and 58 unknown objects. Special attention must be paid to variable WDs as ZZ Ceti-type objects may appear among them.

\section{Conclusions}

POSS1/POSS2 photometry for over 1 billion objects provides a means to examine the objects for variability when deriving the most accurate magnitudes possible from POSS1 and POSS2 measurements. By applying such a method we have revealed 336 variables in the catalogue of FBS blue stellar objects; they include blazars, cataclysmic variables, white dwarfs and other recognized types. A similar approach is being applied to the FBS late-type stars (among which Mira type and semi-regular variables dominate), the Second Byurakan Survey (SBS) objects (blazars, QSOs/Seyferts, cataclysmic variable, white dwarfs, etc.), and to the AGN catalogue for blazars and variable QSOs.

\section{References}

Abazajian, K. N., et al. 2009, ApJS, 182, 543

Cabanela, J. E., et al. 2003, PASP, 115, 837

$\mathrm{H} \varnothing \mathrm{g}, \mathrm{E} .$, et al. 2000, A\&A, 355, L27

Lasker, B., et al. 2008, The GSC, V. 2.3.2, Astron. J., 136, 735

McMahon R. G., Irwin, M. J., \& Maddox, S. J. 2000, APM-North Catalogue (Cambridge, UK: IoA)

Mickaelian, A. M. 2008, AJ, 136, 946

Mickaelian, A. M. \& Sinamyan, P. K. 2010, MNRAS, 407, 681

Mickaelian, A. M., Mikayelyan, G. A., \& Sinamyan, P. K. 2011, MNRAS, 415, 1061

Monet D., et al. 1998, USNO-A V2.0, USNO Flagstaff St. $E$ Univ. Space Res. Ass.

Monet, D. G., et al. 2003, AJ, 125, 984

Samus N. N., et al. 2010, Combined GCVS (Vizier catalog II/250, version 2010)

Wozniak, P. R., Vestrand, W. T., \& Akerlof, C. W. 2004, AJ, 127, 2436 